\title{
Active Vibration Isolation Control: comparison of Feedback and Feedforward control strategies applied to Coriolis Mass-Flow Meters
}

\author{
L. van de Ridder ${ }^{1}$, W.B.J. Hakvoort ${ }^{2}$ and J. van Dijk ${ }^{3}$
}

\begin{abstract}
In this paper we describe the design, implementation and results of multi degree of freedom (DOF) active vibration control for a Coriolis mass-flow meter (CMFM). Without vibration control, environmental vibrational disturbances results in nanometre movement of the fluid-conveying tube which causes erroneous mass-flow measurements. In order to reduce the transmissibility from external vibrations to the internal tube displacement active vibration control is applied.

A comparison of a feedback control strategy (adding virtual mass and skyhook damping) and an adaptive feedforward control strategy is made, taking into account the sensor noise levels. Theoretic results are validated with a multi-DOF experimental setup, showing up to $40 \mathrm{~dB}$ reduction of the influence of external vibrations. The amount of reduction is limited by the sensor noise levels.
\end{abstract}

\section{INTRODUCTION}

A CMFM is an active device based on the Coriolis force principle for direct mass-flow measurements independent of fluid properties [1]. The CMFM contains a fluid conveying tube. An example of a window-shaped tube is depicted in Fig. 1. The tube is actuated to oscillate in resonance with a low amplitude around the $\theta_{\text {twist }}$-axis. A fluid flow in the vibrating tube induces Coriolis forces, proportional to the mass-flow $\dot{\Phi}_{m}$ :

$$
F_{\text {cor }}=-2 L \cdot \dot{\theta}_{t w i s t} \times \dot{\Phi}_{m}
$$

this force results in a rotation around the $\theta_{\text {swing-axis and }}$ thus affecting the modeshape of the actuation mode. Measuring the tube displacements allows measuring the massflow. Besides an effect of the mass-flow on the modeshape, support excitations can introduce motions that cannot be distinguished from the Coriolis force induced motion, thus introducing a measurement error [2], [3]. To reduce the sensitivity of the flow measurement to external vibrations, passive or active vibration isolation can be used. Passive isolation consists of several stages of mass-spring-damper systems between the floor and the casing of a machine [4]. The parameters are adjusted to achieve high-frequency attenuation. However, the performance of passive isolation, applied to a CMFM, is limited [5]. An alternative and widely used approach is to apply active vibration isolation control.

${ }^{1}$ L. (Bert) van de Ridder is with Faculty of Engineering Technology, University of Twente, 7500 AE Enschede, The Netherlands l. vanderiddereutwente.nl

${ }^{2}$ Wouter B.J. Hakvoort is the with Faculty of Engineering Technology, University of Twente and DEMCON Advanced Mechatronics, Enschede, The Netherlands wouter.hakvoortedemcon.nl

${ }^{3}$ Johannes van Dijk is with Faculty of Engineering Technology, University of Twente, The Netherlands j.vandijk@utwente.nl

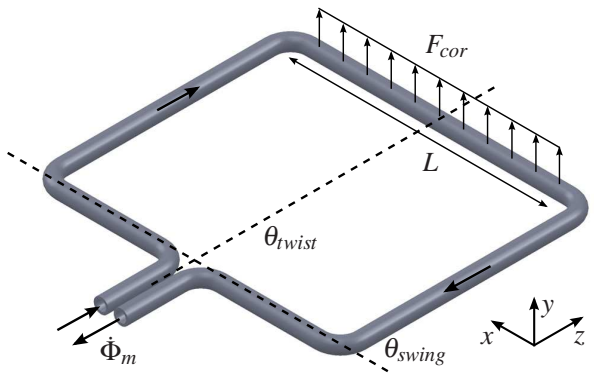

Fig. 1. Window-shaped tube

This paper extends self-tuning feedforward control, presented in [6], and compares it to a feedback strategy [5], both applied to a CMFM. These strategies are compared on the ability to reduce the influence of external vibrations on the mass-flow measurement value. The experimental setup is explained in [6], [7]. Several advances are made: the feedforward strategy is extended to MIMO and the influence of the sensor noise levels is included in the analysis.

The paper starts with a model description in section II. In section III, the different control strategies are presented. Results of the experimental validation are presented in section IV. The paper finalises with a discussion in section V and a conclusion in section VI.

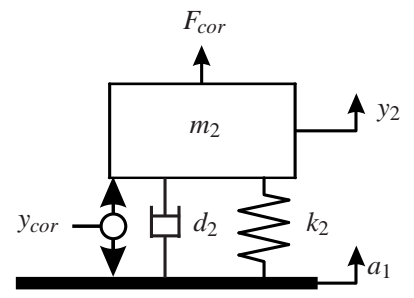

Fig. 2. Simplified model of the CMFM tube [3]

\section{MODEL}

In this section a simplified model is presented. In [3] a complex model of a CMFM is reduced to a 1D mass-spring system. This model is depicted in Fig. 2 and describes the influence of external vibrations $a_{1}$ and the Coriolis effect $F_{c o r}$ (Eq. 1) on the displacement of the tube. From which the mass-flow measurement is derived. The tube properties are $m_{2}=2.45 e-5 \mathrm{~kg}, d_{2}=1.60 e-5 \mathrm{Ns} / \mathrm{m}$ and $k_{2}=10.4 \mathrm{~N} / \mathrm{s}$, resulting in a relative undamped resonance frequency $\omega_{2}=$ $103.7 \cdot 2 \pi \mathrm{rad} / \mathrm{s}$. The actuation mode, a oscillation around the $\theta_{\text {twist }}$-axis, has a resonance frequency $\omega_{\text {act }}=175 \cdot 2 \pi \mathrm{rad} / \mathrm{s}$, 
but this mode is not included in this simplified model. This actuation mode induces the force $F_{c o r}$ at the actuation frequency. The displacement $y_{c o r}$, expressed in the Laplace domain, is equal to:

$$
y_{\text {cor }}(s)=\frac{-1}{s^{2}+\frac{d_{2}}{m_{2}} s+\frac{k_{2}}{m_{2}}} a_{1}(s)+\frac{-1}{m_{2} s^{2}+d_{2} s+k_{2}} F_{c o r}(s)
$$

this displacement is dependent on the external vibrations $a_{1}$ and on the mass-flow $\dot{\Phi}_{m}$, generating $F_{c o r}$.

An external disturbance with a frequency content around the actuation frequency has a direct influence on the massflow measurement value [3]. The transmissibility from $a_{1}$ to $y_{\text {cor }}$ describes this influence. Minimising this transmissibility, without affecting the transfer function of $F_{c o r}$ to $y_{c o r}$, results in a reduction in the sensitivity for external disturbances. The attenuation is only needed in a relatively small $(50 \mathrm{~Hz})$ frequency band around the actuation frequency. This is indicated by a $50 \mathrm{~Hz}$ wide region of interest (RoI) in all the figures in this paper.

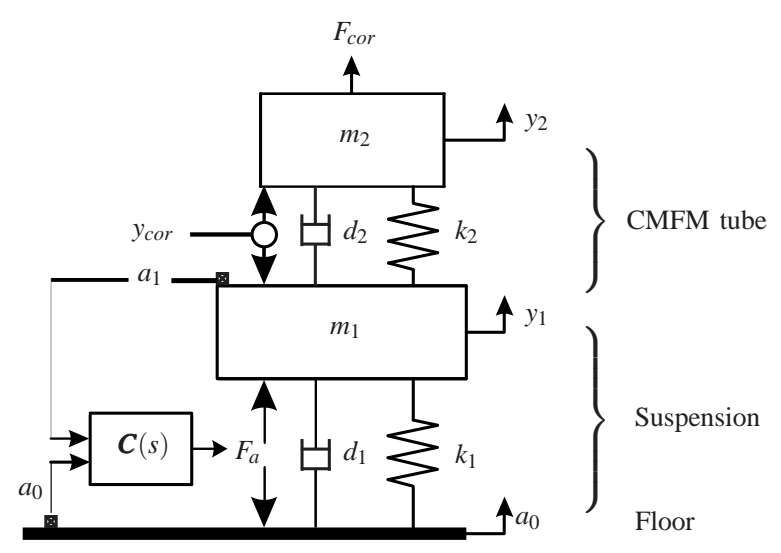

Fig. 3. Mass-damper-spring model: 1D representation of multi-DOF system

A possible solution is to add a passive suspension between de CMFM tube and the floor. This results in an extension of Fig. 2 as depicted in Fig. 3. The influence of external vibrations on the newly introduced stage is:

$$
a_{1}(s)=\frac{d_{1} s+k_{1}}{m_{1} s^{2}+d_{1} s+k_{1}} a_{0}(s)+\frac{s^{2}}{m_{1} s^{2}+d_{1} s+k_{1}} F_{a}(s)
$$

where the effect of the tube on the suspension is neglected, which is only valid if $m_{2} \ll m_{1}$. When we neglect the force $F_{a}$, this is a form of passive vibration isolation [4]. The performance is insufficient, because the suspension frequency is limited by the maximum stress in the connection tubes and a maximum allowable sag due to gravity. Therefore sensors and actuators are added to the model for active vibration isolation control (AVIC). Two realisations of such AVIC stage are given in [5] and [7]. In our model we choose a suspension mode with a resonance frequency of $\omega_{1}=30 \cdot 2 \pi \mathrm{rad} / \mathrm{s}$. For $m_{1}=0.2 \mathrm{~kg}$ and relative damping $\zeta=0.01$, this results in $d_{1}=0.75 \mathrm{Ns} / \mathrm{m}$ and $k_{1}=7.1 e 3 \mathrm{~N} / \mathrm{s}$.

The actuator is operated in voltage-mode in order to obtain the least amount of actuator noise, resulting in an addition pole in the transfer function, which is dependent on the motor-constant $k_{m}$, induction $L$ and resistance $R$ of the actuator coil:

$$
F_{a}(s)=\frac{k_{m}}{L s+R} U(s) \approx \frac{\omega_{\text {ind }}}{s+\omega_{\text {ind }}} U(s)
$$

whereby the low frequency gain is assumed to be 1 .

The model for control can be summarised as follows. A primary path, also called the transmissibility:

$$
P(s)=\frac{a_{1}(s)}{a_{0}(s)}=\frac{d_{1} s+k_{1}}{m_{1} s^{2}+d_{1} s+k_{1}}
$$

and a secondary path, the actively controlled part:

$$
S(s)=\frac{a_{1}(s)}{U(s)}=\frac{s^{2}}{m_{1} s^{2}+d_{1} s+k_{1}} \cdot \frac{\omega_{\text {ind }}}{s+\omega_{\text {ind }}}
$$

both resulting in an acceleration $a_{1}$, which needs to be minimised to reduce the influence on the mass-flow measurement.

\section{CONTROL ALGORITHM}

Actively reducing the influence of external vibrations can be done in several ways. In this section a feedback and an adaptive feedforward strategy are compared on the ability to reduce the transmissibility and to handle sensor noise. For clarity, the model and the control strategies are presented SISO. Only the adaptive algorithm is presented in MIMO, since this is not straightforward.

\section{A. Feedback}

Reconsider the model in Fig. 3. The transmissibility from external vibrations $a_{0}$ to the Coriolis displacement $y_{\text {cor }}$ gives the influence of external vibrations on the measurement value of a CMFM [3]. In [5] and in more detail in [10], a strategy is presented to use acceleration feedback to add virtual mass and skyhook damping to $m_{1}$. This results in a lower suspension frequency and thus a lower transmissibility. The expression for the controller is given by:

$$
\begin{aligned}
C_{F B}(s) & =\frac{U(s)}{a_{1}(s)} \\
& =\left(K_{a}+\frac{K_{v}}{s}\right) \underbrace{\frac{s^{2}}{s^{2}+2 \zeta_{f} \omega_{f} s+\omega_{f}^{2}}}_{H_{1}(s)} \underbrace{\frac{\omega_{r}^{2}}{s^{2}+2 \zeta_{r} \omega_{r} s+\omega_{r}^{2}}}_{H_{2}(s)}
\end{aligned}
$$

where $K_{a}=0.17$ is the added virtual mass and $K_{v}=20.5$ the added skyhook damping to lower and damp the suspension mode with frequency $\omega_{1}$. The definitions of $K_{a}$ end $K_{v}$ are presented in [5]. The term $H_{1}(s)$ is a second-order highpass filter with a corner frequency $\omega_{f}=1 \cdot 2 \pi \mathrm{rad} / \mathrm{s}$ and $\zeta_{f}=0.7$, used to prevent actuator saturation. The term $H_{2}(s)$ is an sightly damped second-order low-pass filter at the tube actuation frequency $\omega_{r}=175 \cdot 2 \pi \mathrm{rad} / \mathrm{s}$. This filter limits the control bandwidth and adds extra attenuation in the region of interest due to a low $\zeta_{r}=0.07$.

The strategy is depicted schematic in Fig. 4 and the result is depicted in Fig. 8, showing an attenuation of the transmissibility in the region of interest. The strategy has disadvantages: High performance requires a high controller 


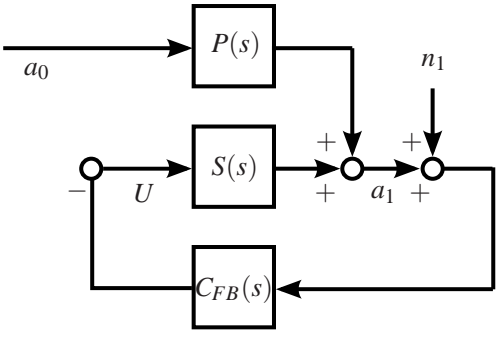

Fig. 4. Feedback scheme, based on (3) and (7). Noise $n_{1}$ is added to the acceleration sensor, measuring $a_{1}$

bandwidth, but the bandwidth is limited by the high frequency dynamics of the system. Therefore, complete knowledge of the system dynamics is required to guarantee a robust and stable system.

\section{B. Adaptive Feedforward}

Alternatively a feedforward strategy can be applied. An extra sensor measures the external vibrations and this signal can be used for compensation of the stage movements. The schematic is depicted in Fig. 5.

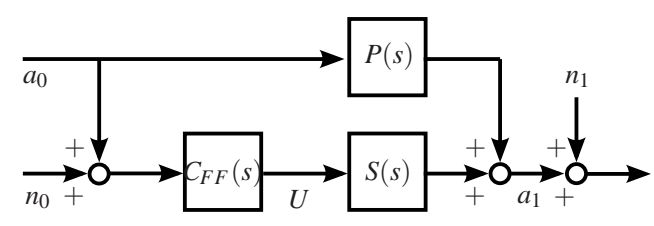

Fig. 5. Feedforward scheme

Optimal compensation can be achieved with the following controller:

$$
C_{F F}(s)=\frac{U(s)}{a_{0}(s)}=-P(s) S^{-1}(s)=-\frac{d_{1} s+k_{1}}{s^{2}} \cdot \frac{s+\omega_{\text {ind }}}{\omega_{\text {ind }}}
$$

Since $P(s)$ and $S(s)$ have poles in common, they cancel out. Therefore, the controller is only dependent on the physical parameters - stiffness, damping and actuator dynamics between the floor and the suspended stage, no knowledge of the internal dynamics is needed. This can be understood conceptually as follows; by compensating the forces due to the stiffness and damping, no forces are transmitted from the floor to $m_{1}$. The controller (8) can be written as a series of Infinite Impulse Response (IIR) filters with ideal parameters:

$$
C_{F F}(s)=\boldsymbol{F}(s) \boldsymbol{w}=\left[\begin{array}{lll}
\frac{1}{s^{2}} & \frac{1}{s} & 1
\end{array}\right]\left[\begin{array}{c}
-k_{1} \\
-d_{1}-\frac{k_{1}}{\omega_{\text {ind }}} \\
-\frac{d_{1}}{\omega_{\text {ind }}}
\end{array}\right]
$$

In practice, only estimated parameters are available. In this paper we propose a filtered-reference least-mean-square (FxLMS) algorithm with residual noise shaping [8] to update the weights, the scheme is depicted in Fig. 6. The IIR filters with fixed poles, makes the adaptation inherently stable. The FxLMS algorithm is explained in the remaining of this subsection. The algorithm minimises the following quadratic cost function:

$$
J(n)=e^{\prime}(n)^{T} e^{\prime}(n)
$$

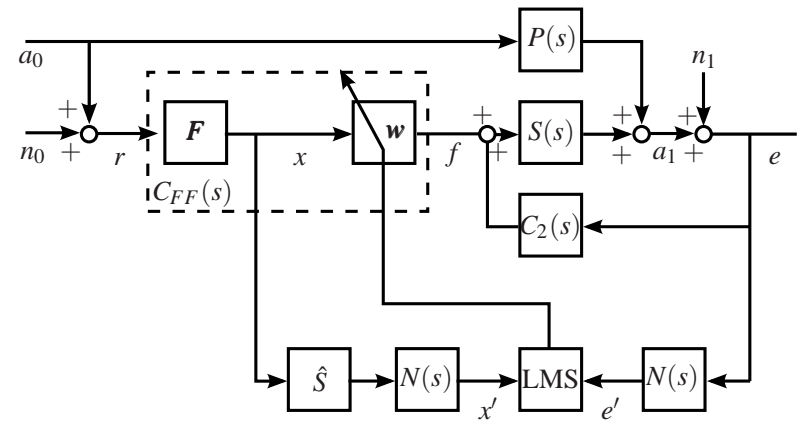

Fig. 6. Modified FxLMS adaptive feedforward control.

with $n$ the iteration step. The filtered error is given by:

$$
e^{\prime}(n)=N e(n)=N(P+S w \boldsymbol{F}) a_{0}(n)
$$

whereby the controller $C_{2}(s)$ is omitted. The error is filtered using the filter $N(s)$ in order to minimise the error in only a small frequency band. $N(s)$ is defined as a $50 \mathrm{~Hz}$ bandpass filter between 150 and $200 \mathrm{~Hz}$ (the region of interest). The weights are determined, using the following update law:

$$
\boldsymbol{w}(n+1)=\boldsymbol{w}(n)-\frac{\mu}{2}\left(\frac{\partial J(n)}{\partial \boldsymbol{w}(n)}\right)^{T}
$$

with adaptation rate $\mu$. For updating the weights, the gradient of the quadratic cost function is needed:

$$
\frac{\partial J(n)}{\partial \boldsymbol{w}(n)}=\frac{\partial J(n)}{\partial e^{\prime}(n)} \frac{\partial e^{\prime}(n)}{\partial \boldsymbol{w}(n)} \approx 2 e^{\prime}(n) N S \boldsymbol{F} a_{0}(n)=2 e^{\prime}(n) \boldsymbol{x}^{\prime}(n)
$$

Merging (12) and (13) gives the update law:

$$
\boldsymbol{w}(n+1)=\boldsymbol{w}(n)-\boldsymbol{\mu} \boldsymbol{x}^{\prime}(n)^{T} e^{\prime}(n)
$$

To determine $\boldsymbol{x}^{\prime}(n)$ the secondary path $S(s)$ is needed. The estimate $\hat{S}$ is a gain matrix, because the gain and phase of $S(s)$ are approximated to be constant in the small frequency band of $N(s)$. Therefore $\boldsymbol{x}^{\prime}(n)$ and $e^{\prime}(n)$ are already aligned in time.

Remaining is the discrete-time implementation of the IIR filter (9). A discrete-time formulation with tame integrators to prevent drift and actuator saturation is proposed.

$$
\boldsymbol{F}(z)=\left[\begin{array}{lll}
\frac{1000 \alpha^{2} T_{s}^{2}}{\left(z-\left(1-\alpha T_{s}\right)\right)^{2}} & \frac{\sqrt{1000} \alpha T_{s}}{\left(z-\left(1-\alpha T_{s}\right)\right)} & 1
\end{array}\right]
$$

where $T_{S}$ is the sample time and the integrators have a cutoff frequency at $\alpha=10 \cdot 2 \pi \mathrm{rad} / \mathrm{s}$. The gains are chosen such that the power of each of the signals in the vector $\boldsymbol{x}^{\prime}(n)$ is equal.

The residual noise filter $N(s)$ tunes the weights such that the transmissibility is minimal in the region of interest. Using the filter has the disadvantage that the suspension frequency is not damped. But damping of this suspension mode is desirable, therefore a simple feedback controller [11] is added to the feedforward strategy:

$$
C_{2}(s)=\frac{U(s)}{a_{1}(s)}=\frac{\omega_{\text {susp }}^{2}}{s^{2}+2 \zeta \omega_{\text {susp }} s+\omega_{\text {susp }}^{2}}
$$


where $\zeta=0.3$ and $\omega_{\text {susp }}=30 \cdot 2 \pi \mathrm{rad} / \mathrm{s} \approx \omega_{1}$ is the suspension frequency. The result of the feedforward strategy is shown in section III-E.

\section{Feedforward MIMO formulation}

The feedforward strategy is presented in SISO, but for the implementation a MIMO formulation is needed, because there are more than one reference and error sensors and multiple actuators in the experimental setup. Therefore the following formulation, introduced by [9], is used:

$$
\begin{aligned}
x_{i, l}(n) & =F_{l} r_{i}(n) \\
x_{i, j, k, l}^{\prime}(n) & =N \hat{S}_{j, k} x_{i, l}(n) \\
\mu(n) & =\frac{\bar{\mu}}{\varepsilon+x_{i, j, k, l}^{\prime}(n) x_{i, j, k, l}^{\prime}(n)} \\
w_{i, j, l}(n+1) & =w_{i, j, l}(n)-\mu(n) x_{i, j, k, l}^{\prime}(n) e_{k}(n) \\
f_{j}(n) & =w_{i, j, l}(n) x_{i, l}(n)
\end{aligned}
$$

where: $\quad I:$ number of reference signals

$J$ : number of actuators

$K:$ number of error sensors

$L$ : number of weights in each filter

\section{Sensor Noise}

Active vibration isolation control is able to reduce the influence of external vibrations. However, every sensor is a possible source for extra disturbances. Therefore, the sensitivity for sensor noise is determined in this section.

Sensor noise is added to the sensors measuring the floor and stage vibrations, respectability $a_{0}$ and $a_{1}$. The noise signals $n_{0}$ and $n_{1}$ are added in the feedback and feedforward strategies in Fig. 4 and 6. For the feedback strategy, the sensitivity is equal to:

$$
S_{F B}=\frac{a_{1}(s)}{n_{1}(s)}=\frac{-S(s) C_{F B}(s)}{1+S(s) C_{F B}(s)}
$$

In the feedforward strategy, there are two sensitivity functions, because there are two sensors:

$$
\begin{aligned}
& S_{F F, 0}=\frac{a_{1}(s)}{n_{0}(s)}=\frac{S(s) C_{F F}(s)}{1+S(s) C_{2}(s)} \\
& S_{F F, 1}=\frac{a_{1}(s)}{n_{1}(s)}=\frac{-S(s) C_{2}(s)}{1+S(s) C_{2}(s)}
\end{aligned}
$$

the sensitivity functions are depending on the secondary path (6) and the designed controllers (Eq. 7,9 and 16). All three sensitivity functions are depicted in Fig. 7. The noise level has a direct influence on the accelerations of the stage:

$$
\Phi_{a_{1}}=\left|S_{a_{1}, n}\right|^{2} \cdot \Phi_{n}
$$

where $\Phi$ is the Power Spectral density of respectively the signals $a_{1}$ and $n$ and $S_{a_{1}, n}$ is one of the above sensitivity functions. For the feedback strategy the sensitivity is much higher in the region of interest, because the feedback tries to compensate for the sensor noise, imposing the stage to

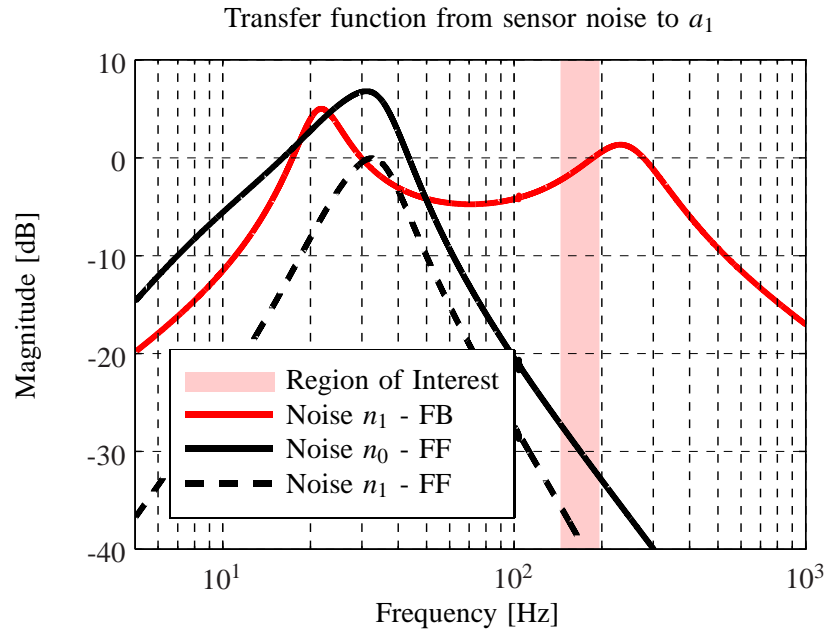

Fig. 7. Sensitivity for sensor noise $(17,18,19)$

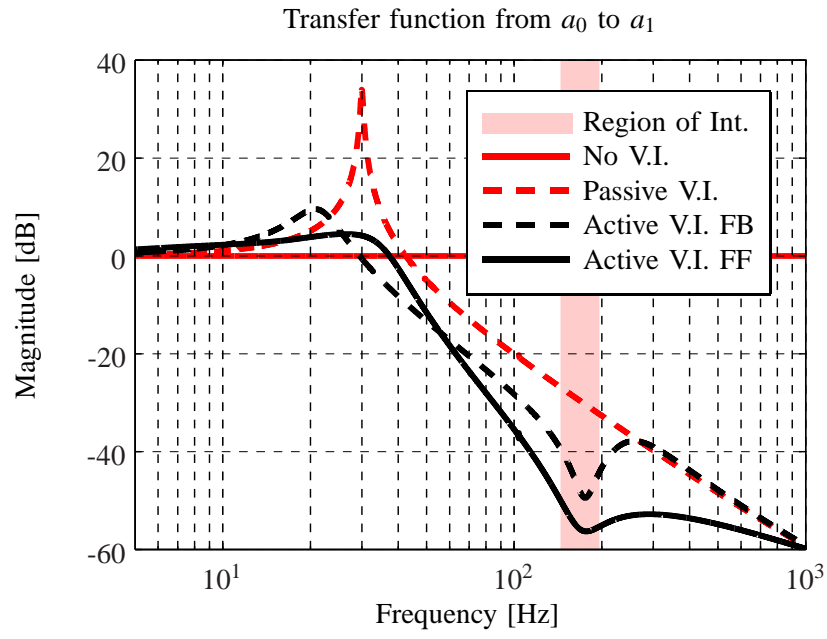

Fig. 8. Modelled transmissibilities of $a_{0}$ to $a_{1}$

move in anti-phase with the noise. Therefore the noise level of the sensors should be much lower than for the feedforward strategies.

\section{E. Model results}

In this section, two active strategies are presented to reduce the influence of external vibrations on the mass-flow measurement value of a CMFM. In Fig. 8 the transmissibility from external vibrations $a_{0}$ to the flexible suspended stage accelerations $a_{1}$ is depicted for all strategies. The best attenuation in the region of interest is achieved using the feedforward strategy, which gives an attenuation of more than $50 \mathrm{~dB}$.

\section{EXPERIMENTAL VALIDATION}

In this section the control algorithm of section III is validated. First the experimental setup is explained. Further the influence of external vibrations is compared for all presented strategies. This is done by the comparing the PSD of the acceleration error signal and the RMS mass-flow measurement error. 


\section{A. Setup}

The experimental setup is depicted in Fig. 9. The details of the modelling and design of the setup are presented in [7]. This CMFM is an active version of the patented design [12].

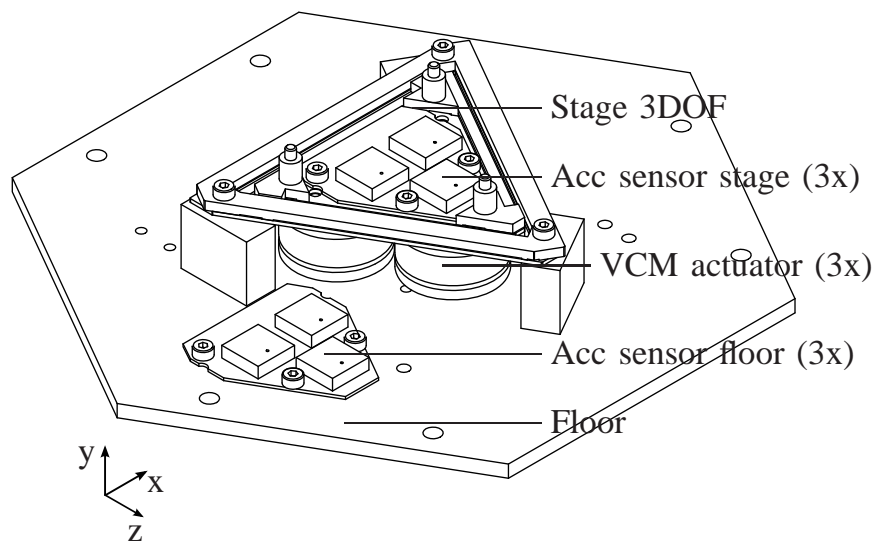

Fig. 9. Solidworks model of the experimental setup [7]. Only the flexible suspended stage is depicted. On top, a Coriolis tube (Fig. 1) is mounted

The used acceleration sensors are Silicon Design 1221$2 \mathrm{~g}$ sensors, selected on their size and noise performance $(5 \mu \mathrm{g} / \sqrt{\mathrm{Hz}})$. Three acceleration sensors on the floor plate are used as reference sensors $\left(a_{0}\right)$.

Because only $y$-translation and $R_{x}$-rotation of the stage are needed to be reduced, see [3], the signals of the three acceleration sensors on the suspended stage are combined to two error signals, containing both the $y$ translation and the $R_{x}$ rotation of the stage. Three voice coil actuators are available to apply a force between the casing and the stage. The active strategies are implemented using MATLAB XPC Target in combination with an NI-6259 data acquisition card. The real-time system runs with a sample rate of $16 \mathrm{kHz}$.

The setup is placed on a 6-sDOF shaker to be able to apply external disturbances. A broadband white disturbance in $y$ direction between 5 and $500 \mathrm{~Hz}$ is applied on the floor plate, for a good comparison of the different configurations.

\section{B. PSD of error sensors}

In Fig. 10 the PSD of both error sensors is depicted for the cases: reference, the passive suspended case, for the feedback strategy and the feedforward strategy. Attenuation in the region of interest is achieved up to $40 \mathrm{~dB}$ with respect to the reference case. It is limited due to the sensor noise, which is $1 e-8\left(\mathrm{~m} / \mathrm{s}^{2}\right)^{2} / \mathrm{Hz}$.

Compared to the passive vibration isolation the active system adds only a minimal extra attenuation. Which is about $10 \mathrm{~dB}$ for both the feedback and feedforward strategy. Outside the region of interest the undamped suspension modes (around $30 \mathrm{~Hz}$ ) and an internal mode (around $300 \mathrm{~Hz}$ ) are clearly visible. Further, harmonics of $100 \mathrm{~Hz}$ are visible, which are probably due to the used voltage source.

\section{Response time}

There are three reference sensors and three actuators, resulting in a 3x3 MIMO controller. Because each direction has three weights (9), there are $3 \cdot 3 \cdot 3=27$ weights in total. The settling of the weights is depicted in Fig. 11 for $\mu=0.0001$. Due to symmetry in the design a couple of weights are approximately the same.

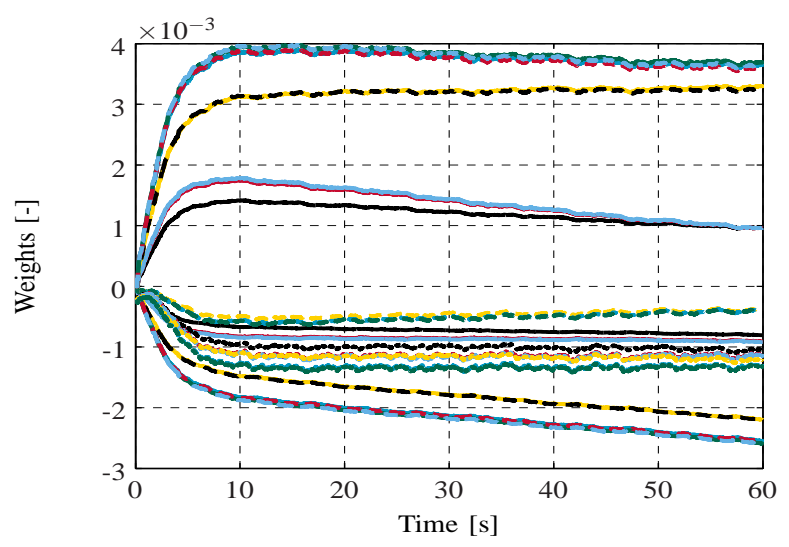

Fig. 11. Time plot of the settling of the weights.

The weights are not a real estimate for the stiffness, damping and induction pool, because these are adapted such that the influence of external vibrations is minimal in the region of interest in the presence of the residual noise shape filter $N(s)$.

TABLE I

RMS MEASUREMENT ERROR VALUES

\begin{tabular}{lcc}
\hline & RMS error [Norm. units] & Reduction [dB] \\
\hline Reference & 0.3043 & 0 \\
Passive & 0.0096 & -30.0 \\
Feedback & 0.0044 & -36.8 \\
Feedforward & 0.0049 & -35.9 \\
\hline
\end{tabular}

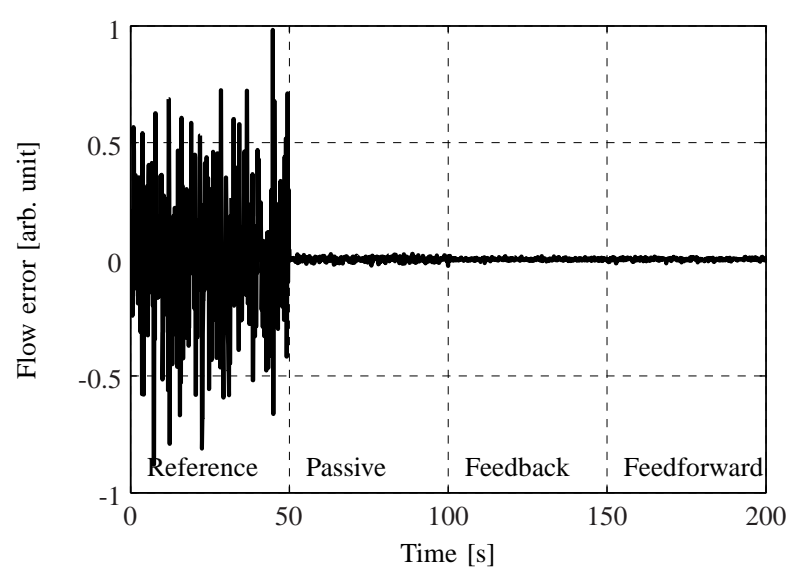

Fig. 12. Time domain RMS flow error (Normalised) - $50 \mathrm{sec}$ of Reference, Passive and Active (feedback and feedforward only) for $a_{0}$ white and broadband disturbance

\section{Measurement Error}

The flexibly suspended stage is build to reduce the influence of external vibrations on the measurement value of a CMFM. Therefore also the newly achieved performance is determined. In Fig. 12 the noise levels are compared for the different configurations. The RMS values are given in 
PSD of error sensor $I$ for $a_{0}$ disturbance

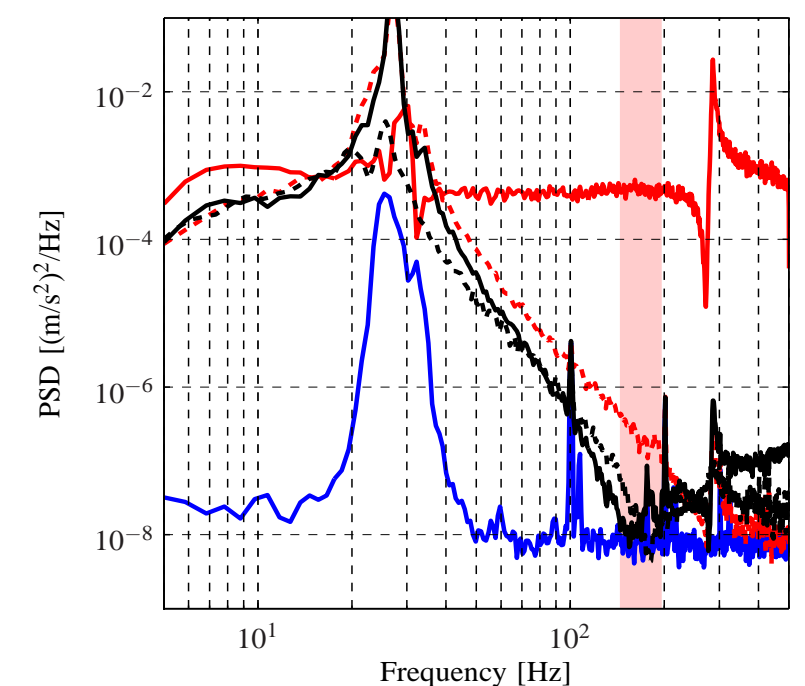

PSD of error sensor $I I$ for $a_{0}$ disturbance

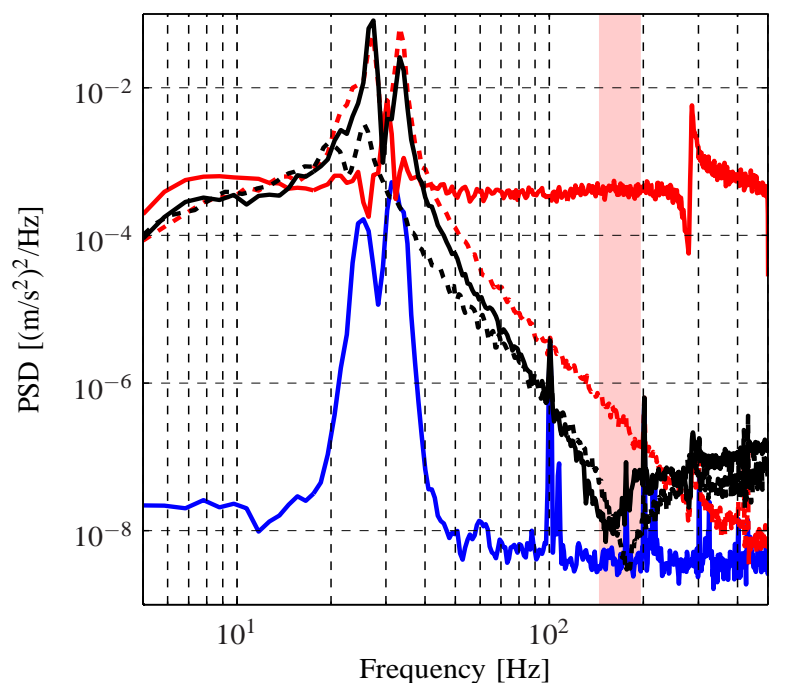

Region of Interest $\longrightarrow$ No Dist. $\longrightarrow$ Ref + Dist.

Passive + Dist. ..... FB + Dist.

FF + Dist.

Fig. 10. PSD of both error sensors $\left(a_{1}\right)$

Table I. Suppression of the RMS measurement error is 36 $\mathrm{dB}$ for both the feedback and feedforward strategy.

\section{DISCUSSION}

Active vibration isolation control is applied to a multiDOF stage. Passive vibration isolation reduces the influence of external vibrations on the measurement value already by $30 \mathrm{~dB}$, control adds another $6 \mathrm{~dB}$ (a factor 2). Based on the transmissibility, this is less than the theoretically expected reduction of up to $50 \mathrm{~dB}$. The performance seems to be limited on both cases by the error sensors, the accelerometers on the stage. For the feedback strategy this is as expected in the noise analyse, see section III-D. The feedforward strategy was expected to perform better, but the tuning of weights is possibly also influenced by the noise level of the error sensors. Instead of using the stage accelerometers, it is better to use the tube displacement sensor $y_{\text {cor }}$.

In the model, the suspension modes of the stage are damped properly. In the experiment, they are still visible due to coupling between the stage modes. Although the undamped modes have a minimal effect on the mass-flow measurement, they should be damped to prevent large tube displacements.

\section{CONCLUSIONS}

Active vibration isolation control, using an adaptive feedforward strategy, showing up to $40 \mathrm{~dB}$ reduction of the influence of external vibrations. It achieves the same performance as the feedback algorithm, but does not suffer from stability issues and is less sensitive for sensor noise. Significant improvements can be made by using better error sensors.

\section{REFERENCES}

[1] M. Anklin, W. Drahm, and A. Rieder, "Coriolis mass flowmeters: Overview of the current state of the art and latest research," Flow Measurement and Instrumentation, vol. 17, no. 6, pp. 317 - 323, 2006.
[2] C. Clark and R. Cheesewright, "The influence upon coriolis mass flow meters of external vibrations at selected frequencies," Flow Measurement and Instrumentation, vol. 14, no. 1-2, pp. 33 - 42, 2003.

[3] L. Van De Ridder, W. Hakvoort, J. Van Dijk, J. Lötters, and A. de Boer, "Quantification of the influence of external vibrations on the measurement error of a coriolis mass-flow meter," Flow Measurement and Instrumentation, vol. 40C, pp. 39-49, 2014.

[4] E. I. Rivin, Passive Vibration Isolation. New York: ASME Press, 2003, iSBN 079810187X.

[5] L. Van De Ridder, M. Beijen, W. Hakvoort, J. Van Dijk, J. Lötters, and A. de Boer, "Active vibration isolation feedback control for coriolis mass-flow meters," Control Engineering Practice, 2014.

[6] M. Beijen, J. Van Dijk, W. Hakvoort, and M. Heertjes, "Self-tuning feedforward control for active vibration isolation of precision machines," in 19th World Congress of the International Federation of Automatic Control (IFAC), 2014.

[7] K. Staman, L. Van De Ridder, W. Hakvoort, D. Brouwer, and J. Van Dijk, "A multi-dof active vibration isolation setup for a coriolis massflow meter," in 29th Annual Meeting of the American Society for Precision Engineering, Boston, USA, 9-14 November 2014.

[8] S. M. Kuo and D. Morgan, Active Noise Control Systems: Algorithms and DSP Implementations, 1st ed. New York, NY, USA: John Wiley \& Sons, Inc., 1995.

[9] M. Bouchard and S. Quednau, "Multichannel recursive-least-square algorithms and fast-transversal-filter algorithms for active noise control and sound reproduction systems," Speech and Audio Processing, IEEE Transactions on, vol. 8, no. 5, pp. 606-618, Sep 2000.

[10] D. Tjepkema, J. van Dijk, and H. M. J. R. Soemers, "Sensor fusion for active vibration isolation in precision equipment," Journal of Sound and Vibration, vol. 331, no. 4, pp. 735 - 749, 2012.

[11] A. Preumont, Vibration Control of Active Structures. Springer Netherlands, 2011, ISBN 978-94-007-2032-9.

[12] A. Mehendale, J. C. Lötters, and J. M. Zwikker, "Mass flowmeter of the coriolis type," EU Patent EP 1719 982, 2006.

\section{ACKNOWLEDGMENT}

The authors thank K. Staman for the design and the assembly of the experimental setup. We also thank the industrial partner Bronkhorst High-Tech for many fruitful discussions. This research was financed by the support of the Pieken in de Delta Programme of the Dutch Ministry of Economic Affairs (PID092051). 